\title{
Randomised trial of an asthma self-management programme for adults
}

\author{
Rae M Allen, Michael P Jones, Brian Oldenburg
}

\begin{abstract}
Background - A hospital based, community service asthma education programme for adults to improve asthma knowledge, promote compliance with medication, and reduce morbidity was evaluated.

Methods - The programme was evaluated using a randomised experimental and control group design with repeated measurements over 12 months. A volunteer community sample of 192 respondents was recruited of whom 116 satisfied the inclusion criteria. At the 12 month follow up some data were obtained for all subjects. Intervention subjects attended four $2 \cdot 5$ hour education sessions at weekly intervals. An asthma knowledge questionnaire was administered and compliance was assessed from diary records. Morbidity was assessed retrospectively by questionnaire, prospectively by diary, and objectively by spirometry and serial peak expiratory flow rate monitoring. The adequacy of medical treatment was also assessed. Data were collected at baseline, immediately after the intervention, and at three, six, nine, and 12 months after intervention.
\end{abstract}

Results - Improvements occurred in knowledge and compliance in the intervention group but the impact on morbidity was modest; this was due, at least in part, to the inadequacy of medical treatment.

Department of Health Promotion and Patient Education, Royal North Shore Hospital,

Australia 2065

$\mathrm{R} M$ Allen

Department

of Behavioural

Sciences,

Macquarie University, Australia 2113

M P Jones

School of

Public Health,

Queensland University

of Technology,

of Technology,

B Oldenburg

Reprint requests to Mr R M Allen

Received 7 June 1994

Returned to authors

21 September 1994

Revised version received

3 January 1995

Accepted for publication

9 March 1995
Conclusions - Treatment of asthma should be reviewed and optimised in conjunction with self-management programmes in order to improve health outcomes.

(Thorax 1995;50:731-738)

Keywords: asthma, patient education, evaluation studies.

Good control of asthma symptoms is a realistic goal for most asthmatic patients. Good asthma control requires both good medical treatment and good self-management. If either of these conditions is not met, reduction is unlikely in any of the indices typically used to assess morbidity. Until recently asthma treatment has phylaxis. As a result of this approach, asthmatic individuals have needed considerable knowledge and skills to assess their symptoms accurately and to make the appropriate therapeutic and behavioural responses. In addition, medical regimens are often complex and require skilled use of devices such as metered emphasised symptomatic relief rather than pro- dose inhalers, nebulisers, and peak flow meters. Given the complexity of the task, it is not surprising that inadequacies in self-management have been implicated as contributing to asthma mortality ${ }^{1}$ and morbidity. ${ }^{2}$

In recent years a number of group education programmes for adults with asthma have been developed ${ }^{3-10}$ that focus on improving patient self-management. Randomised controlled trials of the effectiveness of such interventions ${ }^{8-10}$ have shown a significant but modest impact on morbidity. Acquisition of knowledge, compliance with medication, and the adequacy of medical treatment are factors that may influence the health outcome of these strategies. However, data for these factors are not widely reported. Examination of these factors may provide clues as to how to optimise the potential of adult self-management programmes.

We report the randomised controlled evaluation of a community service self-management education programme for adults with asthma. It was hypothesised that the programme would be effective in improving asthma knowledge and compliance with treatment and, where treatment was adequate, improved compliance would result in reduced morbidity. To identify when any treatment effects occurred and how long any such effect lasted, assessments were made before and immediately after the intervention, and three, six, nine, and 12 months later.

\section{Methods}

\section{ADULT ASTHMA EDUCATION PROGRAMME}

The programme was developed and is conducted by the Department of Health Promotion and Patient Education and Department of Thoracic Medicine at Royal North Shore Hospital, Sydney, Australia, a 750 bed university teaching hospital. The programme was initiated in 1983 and an evaluation of the impact of the programme on asthma management knowledge and skills was reported in $1989 .^{5}$

A detailed account of the content and theoretical background of the programme can be found in that report. In brief, groups of 10-12 adult asthmatic patients attended four 2.5 hour educational sessions held at weekly intervals. A family member or friend was encouraged to accompany each participant in the programme. The programme was led by two asthma educators and a respiratory physician attended one session. Small group discussion was used to assess the understanding, priorities, and needs of the participants. Emphasis was placed on developing a supportive setting in which asthma management skills and behaviour could be 
practised and reinforced. Information was presented by means of lectures, audiovisual, and written materials. ${ }^{5}$ Each participant received a programme manual. ${ }^{11}$

The programme content covered areas of: asthma physiology and aetiology; identification and control of triggers; recognition of symptoms and assessment of symptom severity; medications; lifestyle factors; psychosocial factors; inhaler and peak flow meter skills; and monitoring and recording of peak flow rate (PFR).

Although hospital-based, the programme is offered as a community service for which no medical referral is required. The programme is intended to supplement that of the medical care provider and programme participants were advised to return to their usual asthma care provider.

STUDY DESIGN

A randomised controlled trial design was used and the knowledge, skills, compliance with medication, and morbidity of participants were measured before intervention, immediately after intervention, and 12 months later. In order to monitor implementation, compliance and morbidity were also measured three, six, and nine months after intervention.

RECRUITMENT AND ELIGIBILITY

A volunteer community sample of 192 asthmatic adults responded to newspaper advertisements in February and May 1989 calling for adult asthmatics with moderate to severe asthma to volunteer for asthma research. There were no significant differences in response or recruitment rates or group assignment between the recruitment periods. All the respondents were contacted by telephone. The purpose of the study and obligations were explained and eligibility for inclusion in the study was assessed using a standardised interview protocol.

Respondents were included in the study if they were aged 18-65 years, their diagnosis of asthma had been made by a doctor, and they had experienced asthma symptoms rated as moderate to severe according to published consensus guidelines. ${ }^{12}$ Respondents were excluded from the study if they had any potentially confounding conditions or were current tobacco smokers, had quit in the three months prior to the study, or had previously attended any asthma education programme. Adequate English literacy skills were also required. Of the 192 respondents, 116 (60\%) were eligible to participate in the study. The main reasons for exclusion were age $(50 \%)$ and previous asthma education (25\%).

STRATIFICATION AND RANDOM ASSIGNMENT As the sample was drawn from the community, some variation in the standard of medical care was anticipated. Before group assignment the sample was stratified according to peak flow meter ownership as this was considered likely to indicate better asthma management. The eligible respondents were then randomly assigned -58 to the intervention group and 58 to the control group.

\section{PROTOCOL}

The study protocol was approved by the Royal North Shore Hospital medical research and ethics committee and all participants gave their informed consent to take part in the study. Control group participants were offered places in the education programme at the end of the study period.

\section{Baseline assessment}

After recruitment and random assignment to a group all participants attended a baseline assessment session. Separate sessions were held for intervention group and control group members. Participants completed questionnaires to obtain more detailed demographic data and to assess asthma knowledge, beliefs, attitudes, and quality of life. Inhaler and peak flow meter skills were also assessed. Spirometric measurements were performed to obtain objective measures of lung function. The percentage predicted peak flow rate was determined for each subject.

Those members of the intervention group who did not have a peak flow meter were given one and trained in its use. To assess morbidity and compliance, members of both groups kept daily records of symptoms and medication usage for two weeks. All intervention group members and those 26 control group members who had peak flow meters also recorded morning and evening PFR before and after bronchodilator use.

\section{Intervention period}

Following the two week baseline assessment phase the intervention group participants attended the four weekly education sessions of the asthma education programme. Opportunities were available for them to make up any session missed. Following the baseline assessment phase the control group members kept daily diary records for four weeks, corresponding to the period of the education programme for the intervention group. The four week diary record was returned by mail.

\section{Post intervention assessment}

Immediately after the intervention questionnaires were readministered and a further four weeks of diary records were kept. To assess ongoing compliance and morbidity asthma diaries were also kept by members of both groups for four consecutive weeks at three, six, nine, and 12 months following the intervention. All study participants received a reminder letter before each diary keeping period. Diaries were returned by post and a photocopy sent to the participant for their own records.

To assess maintenance of changes in knowledge, attitudes, and subjectively reported morbidity 12 months after the intervention the appropriate questionnaires were readministered, participants were reinterviewed, 
inhaler and peak flow meter skills were reassessed, and spirometric measurements were repeated.

\section{MEASURES}

Asthma general knowledge questionnaire

A 31 item questionnaire was developed to assess knowledge of asthma concepts covered in the education programme. Knowledge questions were marked as either "true", "false", or "not sure". The knowledge score was the total of correct answers. Content and face validity were assessed to be adequate by the respiratory physicians and asthma educators involved in the development of the programme. To assess the discriminant validity of the questionnaire it was completed by 35 adults who did not have asthma and had never been involved in asthma management. The difference between the mean (SD) total score for the "no asthma" group (17.6 (4.5)) and the mean baseline score for study participants $(25 \cdot 1(3 \cdot 1))$ was statistically significant $(\mathrm{p}<0.0001)$.

As an index of reliability the internal consistency of the items was estimated using the Kuder-Richardson 20 coefficient (KR-20) for dichotomous data as 0.56 for the questionnaire completed at baseline and as 0.75 for the questionnaire completed at the 12 month follow up. A separate sample of 20 subjects completed the questionnaire on two occasions two weeks apart. The reproducibility of scores on the questionnaire was examined using the paired $t$ test. The mean score of $24.4(2 \cdot 4)$ for the first completion and $27.9(2.3)$ for the second were not significantly different.

\section{Compliance with medication}

Participants recorded on the asthma diary record form the name and dosage of prescribed asthma medications. They also recorded any other medications they took for asthma. Alongside each recorded medication they kept a daily record of how much medication was taken and the reasons for taking more or less than the prescribed dose. To be classified as "compliant" participants had to have taken at least $90 \%$ of each of their prescribed medications, using the formula of prescribed - taken/prescribed $\times 100$. The proportion of subjects categorised as compliant was calculated for each group.

Strategies to enhance reliability and validity of asthma diary records suggested by Creer and Winder ${ }^{13}$ were employed in diary design and data collection. Data collected were kept to a minimum and were limited to periods of one month during key phases of the study. During the explanation of the diary keeping procedure it was acknowledged that there were many reasons why asthmatic patients do not take all doses of all medications prescribed for their asthma. Study participants were asked to record the reasons why they took more or less than the prescribed dose of any medication to allow difficulties encountered in complying with medication regimens to be identified and strategies developed to overcome them.

\section{Morbidity}

(1) Assessment by interview: information was collected concerning the number of scheduled and unscheduled doctor and hospital visits made and the number of days when asthma had disrupted daily activities in the previous 12 months. "Disruption" was defined as "being confined to bed or a chair because of asthma symptoms". Ratings were made of the frequency of morning wheeze, nocturnal asthma symptoms, and poor response to bronchodilator medications as follows: 0, never; 1, less than monthly; 2, 1-3 times a month; 3 , 1-3 times a week; 4, 4-7 times a week. The reference periods were the 12 months before the study and the 12 month study period.

(2) Spirometric measurements: two Vitalograph compact spirometers (Vitalograph, Buckingham, UK) were used and recalibrated before use. The two asthma educators who performed the spirometric measurements were trained by an experienced respiratory physician who assessed the values obtained. The best of two measures was recorded for each participant.

Participants were asked not to use bronchodilator medications in the four hours before spirometry unless necessary to control symptoms. The $\mathrm{FEV}_{1} / \mathrm{FVC}$ before bronchodilator medication was measured for each participant. Values obtained were categorised as: $\geqslant 80 \%$, normal; $50-79 \%$, mild to moderate airways narrowing; $<50 \%$, severe airways narrowing; and the proportion of subjects in each category was calculated for the two groups.

(3) Assessment of morbidity by diary record: all participants recorded the presence or absence of asthma symptoms during each 24 hour period of diary keeping and the frequency (as a percentage) of symptom occurrence was calculated. Daily symptom severity was rated according to defined rating categories: 0 , no symptoms; 1, symptoms controlled by medication taken more than three hourly; 2, symptoms controlled by an extra dose of bronchodilator medication; 3, bronchodilator medication required 2-3 hourly; 4 , bronchodilator medication required two hourly or less. The frequency of nocturnal symptoms was also calculated, rated as follows: 0 , not woken; 1 , woken once; 2 , woken twice; 3 , woken more than twice.

Daily records were kept of morning and evening PFR before and after administration of bronchodilator using mini-Wright peak flow meters (Boots, Sydney, Australia). The percentage of predicted PFR was calculated for the recorded values. The proportion of each group who recorded a PFR of $<70 \%$ was calculated. Variability in PFR was also calculated using the formula: highest - lowest/highest $\times$ 100 , using the highest and lowest PFR values for each collection period. The median variability in PFR was calculated for both groups.

Adequacy of asthma control and adequacy of medical therapy

To assess the adequacy of asthma control and medical therapy we used the assessment and 
Table 1 Cross sectional and longitudinal effects of education on knowledge scores

\begin{tabular}{|c|c|c|c|c|c|c|c|c|}
\hline & \multicolumn{3}{|c|}{ Intervention group } & \multicolumn{3}{|c|}{ Control group } & \multirow{2}{*}{$\begin{array}{l}\text { Group difference compared } \\
\text { with baseline } \\
p \text { value }\end{array}$} & \multirow{2}{*}{$\begin{array}{l}\text { Cross sectional group } \\
\text { differences } \\
\text { p value }\end{array}$} \\
\hline & $n$ & Median & Interquartile range & $n$ & Median & Interquartile range & & \\
\hline $\begin{array}{l}\text { Baseline } \\
\text { Immediately after intervention } \\
12 \text { months after intervention }\end{array}$ & $\begin{array}{l}56 \\
55 \\
55\end{array}$ & $\begin{array}{l}26 \cdot 0 \\
29 \cdot 0 \\
29 \cdot 0\end{array}$ & $\begin{array}{l}23-27 \\
28-30 \\
27-30\end{array}$ & $\begin{array}{l}57 \\
55 \\
57\end{array}$ & $\begin{array}{l}25 \cdot 0 \\
26 \cdot 0 \\
27 \cdot 0\end{array}$ & $\begin{array}{l}23-27 \\
24-28 \\
25-28\end{array}$ & $\begin{array}{l}- \\
<0.0001 \\
<0.0001\end{array}$ & $\begin{array}{l}\text { NS } \\
<0 \cdot 0001 \\
0 \cdot 0002\end{array}$ \\
\hline
\end{tabular}

Table 2 Mean (SD) cross sectional and longitudinal effects of education on compliance

\begin{tabular}{|c|c|c|c|c|c|c|}
\hline & \multicolumn{2}{|c|}{ Intervention group } & \multicolumn{2}{|c|}{ Control group } & \multirow{2}{*}{$\begin{array}{l}\text { Group difference } 12 \text { months - baseline } \\
\text { Odds ratio }(95 \% \text { CI) }\end{array}$} & \multirow{2}{*}{$\begin{array}{l}\text { Cross sectional group differences } \\
\text { Odds ratio }(95 \% \mathrm{CI})\end{array}$} \\
\hline & $n$ & $\%$ compliant & $n$ & $\%$ compliant & & \\
\hline $\begin{array}{l}\text { Baseline } \\
\text { Immediately after intervention } \\
3 \text { months after intervention } \\
6 \text { months after intervention } \\
9 \text { months after intervention } \\
12 \text { months after intervention } \\
\end{array}$ & $\begin{array}{l}55 \\
55 \\
46 \\
42 \\
43 \\
47\end{array}$ & $\begin{array}{l}70 \cdot 9(39) \\
83 \cdot 6(46) \\
78 \cdot 2(36) \\
83 \cdot 3(35) \\
88 \cdot 3(38) \\
85 \cdot 1(40)\end{array}$ & $\begin{array}{l}52 \\
49 \\
45 \\
35 \\
37 \\
45\end{array}$ & $\begin{array}{l}59 \cdot 6(31) \\
61 \cdot 2(30) \\
64 \cdot 4(29) \\
62 \cdot 8(22) \\
56 \cdot 7(21) \\
53 \cdot 3(24)\end{array}$ & $\begin{array}{l}- \\
1.95(0.85 \text { to } 4.44)(\mathrm{NS}) \\
1.41(0.67 \text { to } 2.97)(\mathrm{NS}) \\
2.05(0.79 \text { to } 5.28)(\mathrm{NS}) \\
4.03(1.27 \text { to } 12.78)(\mathrm{p}<0.02) \\
3.4(1.22 \text { to } 9.46)(\mathrm{p}<0.02)\end{array}$ & $\begin{array}{l}1.73(0.78 \text { to } 3.84)(\mathrm{NS}) \\
3.41(1.37 \text { to } 8.47)(\mathrm{p}<0.01) \\
2.11(0.84 \text { to } 5.3)(\mathrm{NS}) \\
2.95(1.02 \text { to } 8.55)(\mathrm{p}<0.05) \\
6.15(1.99 \text { to } 19.06)(\mathrm{p}<0.002) \\
5.24(1.95 \text { to } 14.09)(\mathrm{p}=0.001)\end{array}$ \\
\hline
\end{tabular}

therapy criteria suggested in the report of an international panel of asthma specialists. ${ }^{14}$ For each participant in the study asthma severity was rated according to the report criteria. The severity ratings are based on $\mathrm{FEV}_{1}$, and the symptoms and inhaled $\beta_{2}$ agonist use that was recorded in the asthma diaries. Prescribed medical treatment was then compared with the level of medical treatment advised for the rated asthma severity. Medication was rated as "adequate" if the prescribed medication was at least that recommended for the level of symptoms recorded. The occurrence of symptoms would indicate non-compliance with adequate treatment. Medication was rated as "inadequate" if the recorded treatment was less than that recommended for the severity of symptoms recorded. The use of these severity and treatment criteria allowed a rating algorithm to be objectively applied.

\section{STATISTICAL ANALYSIS}

To test whether participation in the programme resulted in significant sustained improvements in compliance and morbidity required repeated measurements on each subject to be analysed. The generalised estimating equations method ${ }^{15}$ using a binomial link function was used in the analysis of dichotomous variables. A subject effect was included in the ordinal regression model for ordered categorical outcomes. In each case the model included main effects of treatment and time, as well as their interaction. The latter provided a test of the homogeneity of treatment effect during the follow up period as well as a comparison of the study groups with respect to longitudinal changes in a given measure. When appropriate, post hoc analyses were performed to compare the group difference at the relevant time points with that at baseline - that is, to compare the treatment effect (for example, odds ratio at follow up) with that at baseline.

Similarly, differences between the groups at each time point were examined to identify where significant changes had occurred. For the cross sectional comparisons of groups dichotomous outcome measures were analysed by unconditional logistic regression and ordered categorical outcome measures by or- dinal regression. None of the quantitative measures was normally distributed and none could be normalised. Consequently, for quantitative outcome measures the Wilcoxon rank sum test was used to test for differences between groups and medians and interquartile ranges are reported. Proportions are expressed as percentages and the odds ratios and $95 \%$ confidence intervals (CI) are reported.

Because of the number of analyses being performed the statistical significance level was defined as $\mathrm{p}<0.01$. Analyses were performed using the programme SPIDA. ${ }^{16}$

\section{Results}

PARTICIPATION AND RESPONSE RATES

Three subjects (two in the intervention group and one in the control group) were withdrawn from the study before the immediate postintervention assessment. One member of the intervention group developed a confounding medical condition and the other moved overseas before completing the education programme. The control group member withdrew because of family health reasons. All remaining 113 subjects were followed up. Re-interview and return rates for questionnaires were close to $100 \%$ (table 1). Return rates for diaries were more variable (table 2). Furthermore, some diary data were not included in the analyses because they were incomplete or, in the case of morbidity data, oral steroid medications were being taken for acute asthma or some other condition. The sample size for each calculation is shown.

\section{DEMOGRAPHIC DETAIIS OF PARTICIPANTS}

There was an approximately equal proportion of men (46\%) and women (54\%) in the sample. The average age was 40 years (range 19-63). Most (93\%) were Australian born or had English-speaking backgrounds; $28 \%$ had never married and $65 \%$ were married or in de facto relationships. Nearly half (49\%) of the subjects had 12 or more years of education, only $4 \%$ had six years or less. There were no significant differences between the groups on any of the demographic variables measured. 

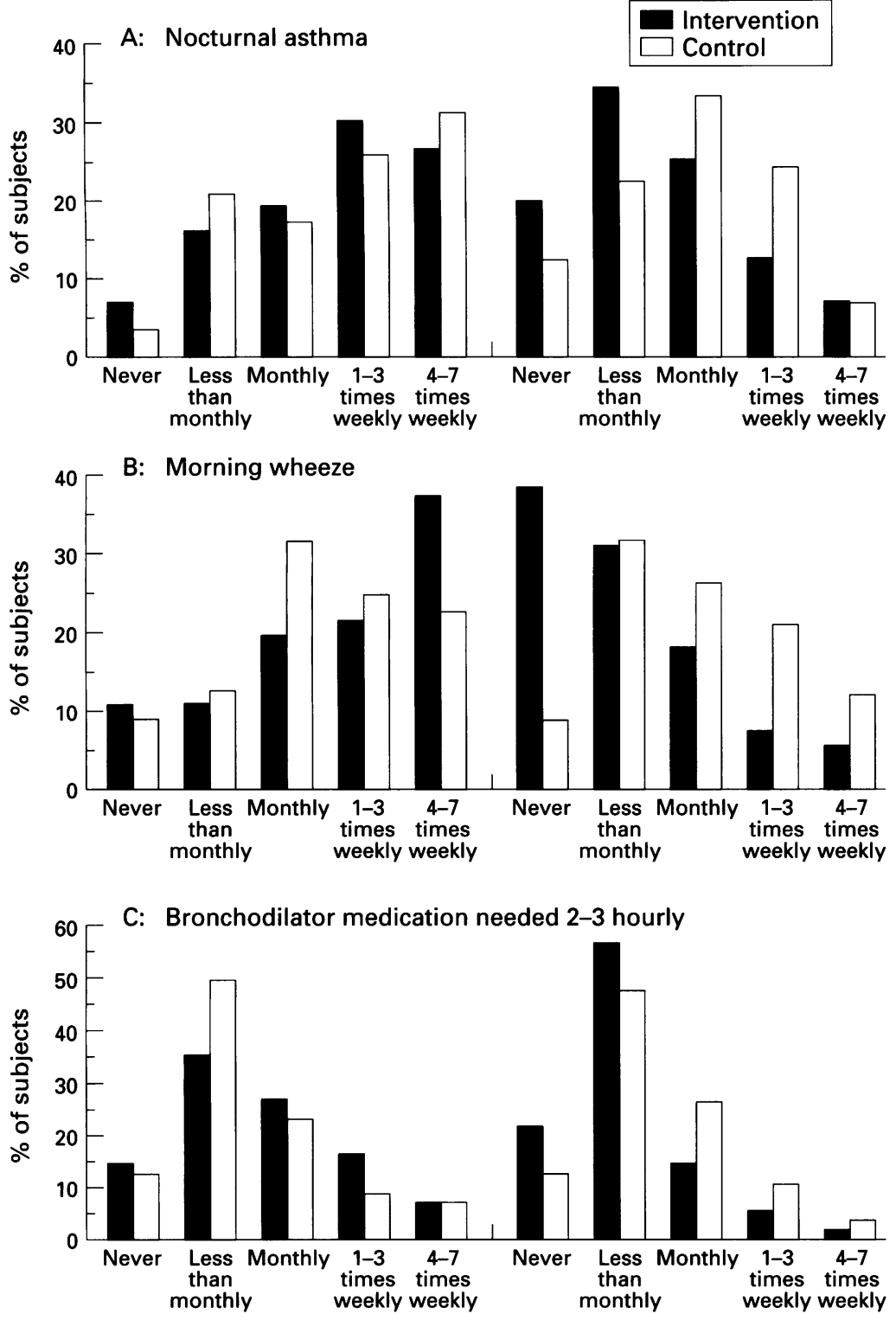

Baseline

12 months after intervention

Perceived morbidity at baseline and 12 months after intervention.

(A) Nocturnal asthma: odds ratio (95\% confidence interval) for group difference between baseline values and 12 months after intervention $0.40(0.85$ to 1.11) $(p=0.08)$; cross sectional group difference 12 months after intervention 1.93 ( 0.99 to 3.78$)(p=0.05)$.

(B) Morning wheeze: group difference between baseline values and 12 months after intervention $0.08(0.03$ to 0.24$)(p<0.0001)$; cross sectional group difference 12 months after intervention $4.02(1.98$ to 8.18$)(p=0.0001)$.

(C) Bronchodilator medication needed 2-3 hourly: group difference between baseline values and 12 months after intervention 0.25 $(0.09$ to 0.71$)(p<0.01)$; cross sectional group difference 12 months after intervention 2.38 (1.15 to 4.94$)(p<0.02)$.

\section{KNOWLEDGE}

From table 1 it can be seen that general asthma knowledge was good for both groups at entry to the study. Compared with the group difference at baseline, the immediate post-intervention difference was statistically significant with those in the intervention group significantly improving their asthma general knowledge. This improvement was maintained for the members of the intervention group 12 months after the intervention. Patients in the control group became more knowledgeable over time but the change from baseline was significantly greater for patients in the intervention group at both assessment points.

\section{COMPLIANCE WITH MEDICATION}

At baseline $65 \%$ of subjects were compliant with prescribed medical treatment (table 2). The group $\times$ time interaction was not significant. However, the comparisons of odds ratios during follow up with those at baseline, although of marginal statistical significance, suggest that the significant differences between the groups immediately after intervention and nine and 12 months later are not simply reflections of pre-existing baseline differences between the groups. The odds ratios comparing the two study groups at each assessment indicate that the odds of compliance in the intervention group are always greater than for the control group, varying from twofold to sixfold. This is particularly pronounced towards the end of the follow up period when the odds ratios were 5-6 fold.

\section{MORBIDITY}

There were no significant differences between the groups at baseline for any measure of morbidity, nor were there any significant baseline differences within either group for any morbidity measure for those groups members who had a peak flow meter at entry to the study compared with those who did not.

In the 12 months before the study little disruption to daily activities or need to seek acute asthma care was reported (table 3 ). Small reductions occurred in these measures over the study period for both groups and the group differences were not significant. Participants in the programme were encouraged to seek regular review of asthma, even when asymptomatic. For both groups the median of four consultations for asthma review (interquartile range 2-7) 12 months after the intervention was not significantly different from that at baseline.

Table 3 Morbidity in the 12 months before the study and adequacy of medical treatment at baseline

\begin{tabular}{|c|c|c|}
\hline & $\begin{array}{l}\text { Intervention } \\
\text { group }\end{array}$ & $\begin{array}{l}\text { Control } \\
\text { group }\end{array}$ \\
\hline \multicolumn{3}{|l|}{ (a) Retrospective interview } \\
\hline \multicolumn{3}{|c|}{ No. of days activities disrupted by asthma: } \\
\hline Interquartile range & $0-9$ & $0-6$ \\
\hline No. of subjects & 56 & 57 \\
\hline \multicolumn{3}{|c|}{ Unscheduled use of medical services for acute care: } \\
\hline Median & 2 & 1 \\
\hline Interquartile range & $1-3$ & $0-2$ \\
\hline No. of subjects & 56 & 57 \\
\hline \multirow{2}{*}{\multicolumn{3}{|c|}{$\begin{array}{l}\text { (b) Prospective diary assessment } \\
\text { Percentage of days asthma symptoms recorded: }\end{array}$}} \\
\hline & ns recorded: & \\
\hline Median & $71 \cdot 0$ & $50 \cdot 0$ \\
\hline Interquartile range & $29-100$ & $14-86$ \\
\hline No. of subjects & 55 & 53 \\
\hline \multicolumn{3}{|c|}{ Percentage of nights asthma symptoms recorded: } \\
\hline Median & $14 \cdot 0$ & $11 \cdot 0$ \\
\hline Interquartile range & $0-57$ & $0-43$ \\
\hline No. of subjects & 55 & 53 \\
\hline \multicolumn{3}{|c|}{ Percentage reporting PFR $<70 \%$ predicted: } \\
\hline & $70 \cdot 0$ & $65 \cdot 0$ \\
\hline $\begin{array}{l}\text { No. of subjects } \\
\text { Peak flow lability: }\end{array}$ & 55 & 26 \\
\hline \multicolumn{3}{|l|}{ Peak flow lability: } \\
\hline $\begin{array}{l}\text { Median } \\
\text { Interquartile range }\end{array}$ & $58 \cdot 4$ & $48 \cdot 3$ \\
\hline Interquartile range & $35 \cdot 0-77 \cdot 0$ & $37 \cdot 0-75 \cdot 0$ \\
\hline $\begin{array}{l}\text { No. of subjects } \\
\text { (c) FEV,/FVC }\end{array}$ & 54 & 26 \\
\hline \multicolumn{3}{|l|}{ (c) $\mathrm{FEV}_{1} / \mathrm{FVC}$} \\
\hline Normal $(\geq 80 \%)$ & & $10 \%$ \\
\hline Mild/moderate (79-50\%) & $77 \%$ & $78 \%$ \\
\hline Severe $(<50 \%)$ & $7 \%$ & $12 \%$ \\
\hline $\begin{array}{l}\text { No. of subjects } \\
\text { (d) Adequacy of medical treatment }\end{array}$ & 56 & 57 \\
\hline \multicolumn{3}{|l|}{ (d) Adequacy of medical treatment } \\
\hline No. of subjects & 56 & 53 \\
\hline
\end{tabular}


Although asthma does not appear to have caused much disruption or need for acute care, considerable asthma morbidity was recorded at baseline (table 3, figure). Additionally, in the baseline diaries $19 \%$ of subjects recorded that they had required bronchodilator medication 2-3 hourly to control asthma symptoms and $29 \%$ reported waking more than once nightly with asthma symptoms. Comparison of the odds ratio at 12 months after intervention with that at baseline indicates that the intervention group experienced a greater reduction in the perception of morning wheeze and requirement for bronchodilator medication three hourly or more frequently than did the control group. Otherwise, there were no significant reductions on any of the morbidity measures shown in table 3 between the groups or between the group differences at any time point and that at baseline.

\section{ADEQUACY OF ASTHMA TREATMENT}

At baseline surprisingly few of the study participants had adequate medical treatment to control their asthma as rated according to the published criteria. ${ }^{17}$ At the 12 month follow up assessment point this situation was virtually unchanged, only eight subjects in each group being rated as having adequate treatment. There were too few participants with adequate medical treatment to conduct the planned comparisons of morbidity indices for those compliant with adequate medical treatment and those compliant with inadequate treatment.

\section{Discussion}

The Royal North Shore Hospital asthma selfmanagement education programme for adults has been successful in achieving and maintaining its education objective of improving knowledge about asthma and self-management skills. In addition, the programme aim of sustained improvement in adherence to treatment regimens was achieved. However, despite significant improvements in knowledge and compliance, the overall impact on morbidity was modest. There were significant improvements in perceived morbidity but no delayed or shortlived improvements in objective morbidity measures. The continuing high levels of morbidity are more likely to reflect the inadequacy of medical treatment than deficiencies in study methods.

The study design and methods have a number of strengths. Sample size was clearly adequate in this study as statistically significant differences were found in central outcome variables. The response rates were good and results are likely to be representative of the target population. The measure of asthma knowledge was shown to be reliable and valid. Compliance data were based on prospective diary records and published criteria were used to assess the adequacy of medical treatment. The clinical signs and physiological measures considered characteristic of asthma are neither universal nor unique and the intensity of physiological measures to define asthma and to categorise severity are arbitrary. This lack of precision hampers research design and the interpretation and comparison of findings. We assessed morbidity retrospectively by interview and prospectively by diary record, as well as more objectively by spirometry. Terms and ratings were clearly defined.

The ultimate goal of asthma education strategies is to reduce morbidity. The reduction in perceived morbidity, but not in physiological measures of asthma, found in this study is consistent with other randomised controlled trials. ${ }^{89}$ In this study utilisation of medical services for acute asthma care was low in the 12 months before intervention (table 3 ) and consequently the small reductions that occurred were non-significant.

Utilisation of acute asthma care services is commonly used as an outcome indicator in the evaluation of asthma education interventions. Examination of the findings of this and similar studies suggests that, for samples of asthmatic subjects drawn from the community, recent use of acute care for asthma may motivate participation in programmes but a more extensive follow up period than 12 months may be needed for significant group differences to be detected. The sample of university clinic patients recruited by Bailey and coworkers ${ }^{8}$ had high rates of acute care utilisation at baseline. The group difference was non-significant 12 months after intervention as a dramatic decrease in hospital presentation or admission had occurred for both groups. Wilson and colleagues ${ }^{9}$ found no significant difference in acute care consultations 12 months after intervention, but significant reduction at 24 months in a sample drawn from members of a health care plan.

In contrast, Yoon and coworkers ${ }^{10}$ recruited a sample from patients admitted to hospital for acute asthma care. They reported significant group differences in physiological measures five months after intervention that were not sustained in the longer term. They also showed a dramatic reduction in hospital presentations and admissions. However, as the authors have documented, ${ }^{17}$ participation rates were low and patients of one of the authors were more likely to participate.

Asthma morbidity may be influenced by a variety of factors within and outside the direct control of the programme. Our study allowed us to look at some of these factors - acquisition of knowledge, compliance with treatment regimens, and adequacy of medical treatment. The study participants were knowledgeable at the outset, but programme attendance resulted in a significant and sustained improvement in asthma knowledge. Similarly, for the more committed subjects who continued to participate in the study of Yoon et $a l^{10}$ there was a significant improvement in knowledge of aspects of asthma self-management. However, knowledge is not invariably translated into behaviour.

In the study reported here compliance with medical treatment, assessed after group assignment, was good at baseline for both groups (table 2). Following intervention the odds of 
compliance in the intervention group are always greater than for the control group. It might be argued that compliance was overstated despite strategies to encourage honest reporting. Participants in the study were actively encouraged to record reasons for departures from prescribed treatment and their responses were disarmingly frank. Bailey and colleagues ${ }^{8}$ also realised their programme aim of improved adherence to treatment regimens, reporting substantial improvements in inhaler and medication adherence in their intervention group.

The adequacy of medical treatment is generally assumed in the evaluation of self-management strategies. No matter how compliant the participant, if medical treatment is inadequate there is little likelihood of significant improvement in any of the morbidity indices typically used in assessment of programme effectiveness. A standard protocol was available to guide the medical treatment of participants in the study by Bailey and colleagues. ${ }^{8}$ However, it is not recorded whether this treatment protocol was uniformly and consistently implemented. We adopted a set of criteria ${ }^{14}$ to rate the adequacy of medical treatment irrespective of patient compliance. As the asthma education programme is a community service programme, some variation in the standard of medical treatment was expected. However, nearly $90 \%$ of the study participants were judged to have inadequate medical treatment at baseline and there was virtually no change in this situation during the study period.

The poor ratings of medical adequacy were principally attributable to prescription of regular daily $\beta_{2}$ agonist therapy $(84 \%)$ and inadequate anti-inflammatory medication $(64 \%)$. The previously prevailing view of airways hyperresponsiveness as the primary asthma mechanism had favoured the use of bronchodilator medications for prophylaxis. The acceptance of inflammation as the primary asthma mechanism, increased availability of inhaled anti-inflammatory medications since the late $1980 \mathrm{~s}$, and evidence that long term $\beta_{2}$ agonist therapy may increase airways hyperresponsiveness $^{18}$ has led to the recommendation of anti-inflammatory medications as maintenance treatment for asthma. ${ }^{14}$

Lack of continuity of care because of geographical mobility or seeking treatment only for acute attacks of asthma may have led to inadequate medical treatment. The participants in this study had considerable contact with the medical profession in the 12 months before the study and the 12 months of the study period. Nevertheless, their medical treatment remained suboptimal. Several Australian community based studies of adults with asthma have shown that undertreatment of the condition is as common as $60 \% .^{1920}$

Programme outcomes research can be broadly categorised as efficacy or effectiveness trials. ${ }^{21}$ Efficacy trials are designed to test what a programme achieves under optimum conditions and require committed, motivated subjects and controlled designs. Efficacy trials are considered to be necessary precursors to effectiveness trials that test programme achievements in more real life settings - for example, generalisability trials.

The randomised controlled study reported here approximates the requirements for an efficacy trial. The participation rate and baseline levels of knowledge and compliance indicate a committed, motivated sample. The sample was representative of the area which, according to 1986 census data, ${ }^{22}$ has higher employment rates, average household income, and educational qualifications than either the state or the country as a whole. Under these socioeconomically advantageous conditions the programme has demonstrated efficacy in achieving significant, sustained improvements in knowledge and compliance. The efficacy of the programme in conjunction with optimal medical treatment may need to be demonstrated before effectiveness trials of generalisability are undertaken.

Good asthma control requires not only good self-management but also good medical treatment. To date the focus has been on improving self-management and the adequacy of medical treatment has been neglected. The findings of our study suggest that the adequacy of medical treatment needs to be taken into account when evaluating asthma self-management interventions. To maximise programme usefulness it is also desirable to review and optimise medical treatment in conjunction with the programme.

We thank Ms Gill Browne and Ms Wendy Starr who led the education groups and provided invaluable assistance with the data collection. We gratefully acknowledge Dr David Allen for his helpful comments on the manuscript. The study was partly funded by the NSW Department of Health. Peak flow meters were supplied by Allen and Hanburys.

1 Robertson CF, Rubinfeld AR, Bowes G. Deaths from asthma in Victoria: a 12 month survey. Med f Aust 1990;152 511-7.

2 Horn CR, Clark TJH, Cochrane GM. Compliance with inhaled therapy and morbidity from asthma. Respir Med 1990;84:67-70.

3 Hindi-Alexander MC, Throm J, Middleton E. Collaborative asthma self-management: evaluation designs. Clin Rev Allergy 1987;5:249-58.

4 Snyder SE, Winder JA, Creer TL. Development and evaluation of an adult asthma self-management program: Wheezers Anonymous. F Asthma 1987;24:153-8.

5 Bauman AE, Craig AR, Dunsmore J, Browne G, Allen $\mathrm{DH}$, Vandenberg $\mathrm{R}$. Removing barriers to effective selfmanagement. Patient Education and Counseling 1989;14: 217-26.

6 Worth H. Patient education in asthmatic adults. Lung 1990; Suppl:463-8.

7 Ringsberg KC, Wiklund I, Wilhelmsen L. Education of adult patients at an 'asthma school': effects on quality of life, knowledge and need for nursing. Eur Respir $\mathcal{F} 1990$; 3:33-7.

8 Bailey WC, Richards JM, Brooks CM, Soong S, Windsor RA, Manzella BA. A randomised trial to improve selfmanagement practices of adults with asthma. Arch Intern Med 1990;150:1664-8.

9 Wilson SR, Scamagas P, German DF, Hughes FW, Lulla $S$, Coss S, et al. A controlled trial of two forms of selfmanagement education for adults with asthma. Am $\mathcal{F}$ Med management educat

10 Yoon R, McKenzie DA, Bauman A, Miles DA. Controlled trial evaluation of an asthma education programme for adults. Thorax 1993;48:1110-6.

11 Browne G. Asthma education handbook. Royal North Shore Hospital, Sydney, Australia, 1986.

12 Woolcock AJ, Rubinfeld AR, Seale JP, Landau LL, Antic R, Mitchell C, et al. Thoracic Society of Australia and New Zealand asthma management plan, 1989. Med $\mathcal{F}$ Aust 1989;151:650-3.

13 Creer TL, Winder JA. Asthma. In: Holroyd KA, Creer TL. eds. Self-management of chronic disease. Orlando: Academic Press, 1986;269-303.

14 Hargeave FE, Dolovich J, Newhouse MT. The assessment and treatment of asthma: a conference report. $\mathcal{F}$ Allengy Clin Immunol 1990;85:1098-111.

15 Zeger SL, Liang K-Y. Longitudinal data analysis for discrete and continuous outcomes. Biometrics 1986;42:121-30. 
16 Gebski V, Leung O, McNeil D, Lunn D. SPIDA User's Manual. Version 6. Sydney: Southwood, 1992

17 Yoon R, McKenzie DA, Miles DA, Bauman A. Characteristics of attenders and non-attenders at an asthm education programme. Thorax 1991;46:886-9.

18 Sears MR, Taylor DR, Print CG, Lake DC, Li Q, Flannery $\mathrm{E}$, et al. Regular inhaled beta-agonist treatment in bronchial asthma. Lancet 1990;336:1391-6.

19 Henry DA, Sutherland D, Francis L and the Newcastle Retail Pharmacy Research Group. The use of non-preRetail Pharmacy Research Group. The use of non-pre-
scription salbutamol inhalers by asthmatic patients in the
Hunter Valley, New South Wales. Med f Aust 1989;150: 445-9.

20 Gibson PG, Henry D, Francis L, Cruickshank D, Dupen $\mathrm{F}$, Higginbotham $\mathrm{N}$, et al. Association between availability of non-prescription $\beta_{2}$-agonist inhalers and undertreatment of asthma. BMF 1993;306:1514-8.

21 Flay BR. Efficacy and effectiveness trials (and other phases of research) in the development of health promotion proof research) in the development of health promotion pro-
grammes. Prev Med 1991;15:451-74. 22 grames. Prev Med 1991;15:451-74. Australian Bureau of Statistics. Tables CX1148, CX1155; 1986 Census. 